\title{
Experimental investigation of the effect vertical oscillation on the heat transfer coefficient of the finned tube
}

\author{
S. K. Kadhim ${ }^{1,2, a}$, M. S. Nasif 1 \\ ${ }^{1}$ Mechanical Engineering Department, Universiti Teknologi PETRONAS, 32610 Bandar Seri Iskandar, Perak, Malaysia \\ ${ }^{2}$ Control and System Engineering Department, University of Technology, Baghdad, Iraq
}

\begin{abstract}
The aim of this work is to investigate experimentally the effect of the forced vibrations on the free convection heat transfer coefficient using heated longitudinally finned cylinder made of Aluminium. The effect of the vibration frequency ranged from 2 to $16 \mathrm{~Hz}$ with various heat fluxes ranged from $500-1500 \mathrm{~W} / \mathrm{m}^{2}$. It was found that, the relation between the heat transfer coefficient and amplitude of vibration increased for all inclination angles from $\left(0^{\circ}-45^{\circ}\right)$, while the increment of inclination angle decreases the values of convection heat transfer coefficient. The results show that the heat transfer coefficient ratio $(h v / h o)$ of longitudinal finned cylinders in $\left(0^{\circ}\right)$ angle was $(8 \%)$ and $(30 \%)$ greater than those for the $\left(30^{\circ}\right)$ and $\left(45^{\circ}\right)$ respectively.
\end{abstract}

\section{Introduction}

Extended surface is used to increase the heat dissipation in many engineering and industrial applications by increasing the heat transfer area, also these surfaces used in cooling of electronics, thermo electronic devices, and solar energy applications, cooling of nuclear reactor fuel elements, improving heat transfer in radiators for air conditioning and in air cooled heat exchangers. [1].

Keil and Baird [2] studied the overlap between vibration and free convection heat by vibrating plate in both horizontal and vertical orientation. The study has addressed the effect of both frequency and amplitude of vibration and also took into account the temperature differences in coefficient of vibrated convection heat surfaces.

Kim et al. [3] have conducted an experimental study which shows the effect of vertical mechanical vibrations on the heat transfer rate from cylindrical tubes submerged in a water pool. The diameter of the tube in this study was $(194 \mathrm{~mm})$ and frequency ranging of vibration from $0 \mathrm{~Hz}$ up to $40 \mathrm{~Hz}$ with an amplitude range of (0 to 2.54$) \mathrm{mm}$. They found that low mechanical vibration to have no effect on the heat transfer coefficient which was attributed to the dominance of free convection in this case.However, they found that at $40 \mathrm{~Hz}$ frequency level of vibrations, heat transfer coefficient was increased by $400 \%$

Zaki and El-Kassem [4] studied the external vibration effect on the heat transfer characteristics of single and two-phase flow within the heated annular tube made of stainless steel. The annular tube was connected

${ }^{\text {a }}$ Corresponding author: Saleem.khalefa@gmail.com to a vibrating system with frequency ranged from 0-134 Hz. They concluded that increasing the vibration frequency, lead to decreasing the surface temperature. In case of single-phase flow, it was found that $34 \%$ increase in Nusselt number values was achieved when vibration was implemented.

Previous studies have investigated the effect of vibration on the heat transfer coefficient of cylindrical surfaces and plate. However, to the best of our knowledge, no research has been performed to investigate the effect of vibrations on the fin cylinder, including considering different frequency, heat flux and different inclination angle on the heat transfer coefficient. Therefore, this study investigates the effect of vertical vibration on the finned cylinder, such as vibration intensity, inclination angle and Reynolds number where different heat flux $(500$ to $1500 \mathrm{~W} / \mathrm{m} 2)$ and variable frequency from $(2 \mathrm{~Hz}$ to $16 \mathrm{~Hz}$ ) were used to determine the natural heat transfer coefficient of finned cylinder.

\section{Test rig description}

Two types of cylindrical aluminum finned hot surfaces were used in this experiment which are triangular and rectangular longitudinal fins. The total length of the heated surface is $(320 \mathrm{~mm})$, the finned length is $(300$ $\mathrm{mm}$ ), the outer diameter is $48 \mathrm{~mm}$ at the finned end. The height of the fin is $13 \mathrm{~mm}$. Eight triangular and rectangular longitudinal fin equally distributed by (45o) in both models were used (Fig 1a). A heater is placed inside the hot surface internal diameter and heat flux is varied. Fig. (1a) shows the two heated surfaces. Fig. (1b) 
shows that the holder is U-shaped and made from aluminum substance to hold the weight exerted on vibrator. The holder base is also made of aluminum. The hot surfaces are fixed to connection arms using Teflon substances to reduce the heating loss at its ends. The angle of the hot surface can be changed from $\theta=0^{\circ}$, to $45^{\circ}$ using a lever mechanism which connects the holder and its base. Thereby, the measured vibration amplitude would be approximately the same for both the holder and the heated cylinder. The electric circuit includes a heating coil power of $1000 \mathrm{~W}$. Eight thermocouples were installed at the heated surface and distributed uniformly. Ambient temperature is measured by using a thermocouple which is placed $15 \mathrm{~cm}$ away from the hot surface.

The test was carried out first by heating the hot surface and ensure steady state conditions was achieved after 4 hours. Then temperature reading was recorded with no vibration. Once temperature readings were obtained, the vibration effect was applied and the same procedure to achieve steady state and record the temperature were performed and vibration amplitude was recorded as well. The applied vibration ranged between 2 to $16 \mathrm{~Hz}$.

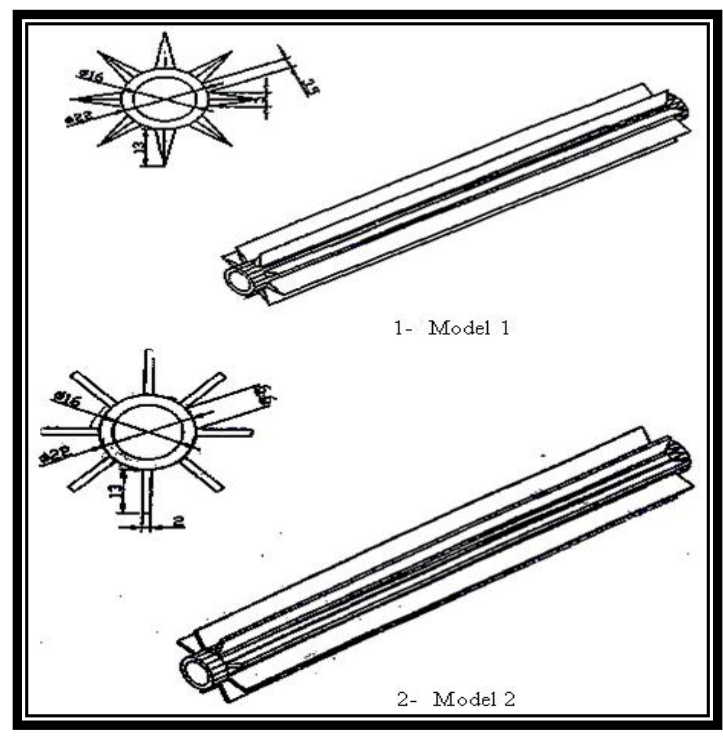

(a)

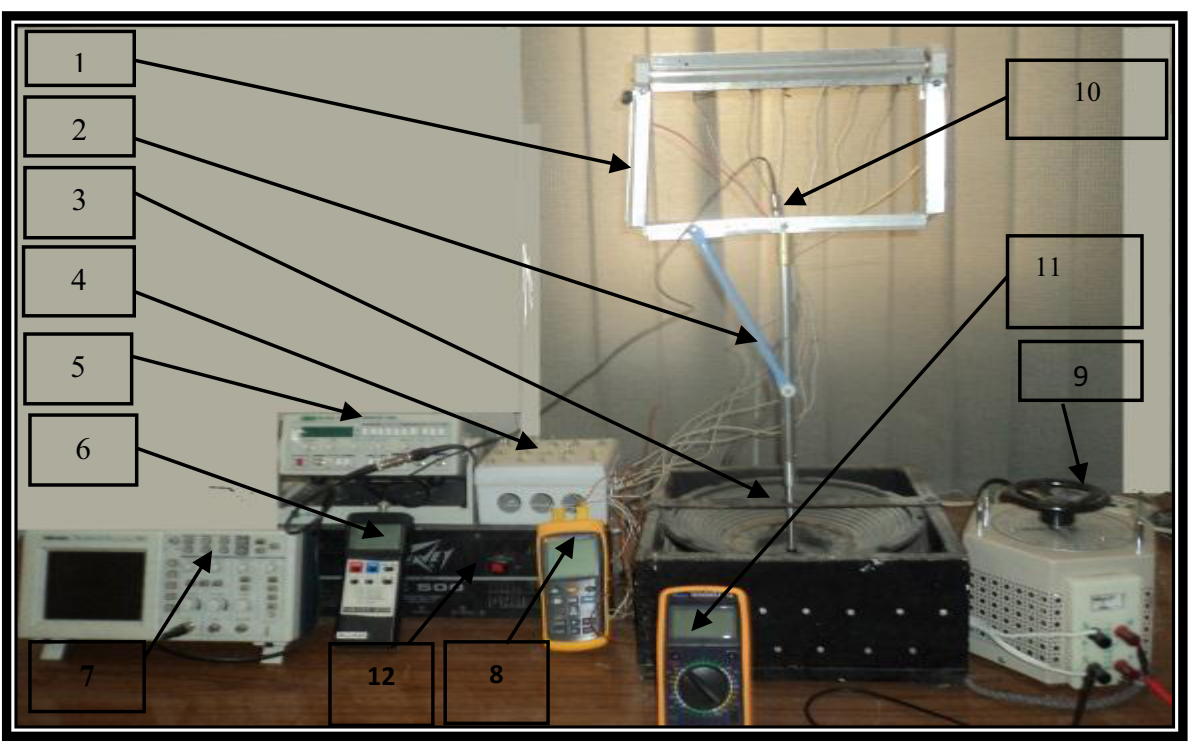

(b)

1-U-shaped, 2- lever mechanism, 3- Vibration Exciter, 4- Selector Switch, 5- Sine Function Generator , 6- Accelerometer , 7- Digital Oscilloscope, 8- Digital Thermometer, 9- Auto transmission, 10- Force Transducer, 11- Digital Voltmeter Ohm Meter Ammeter, 12- Power Amplifier

Figure 1. The photograph shown the experimental rig (Thermal and vibration setup) 


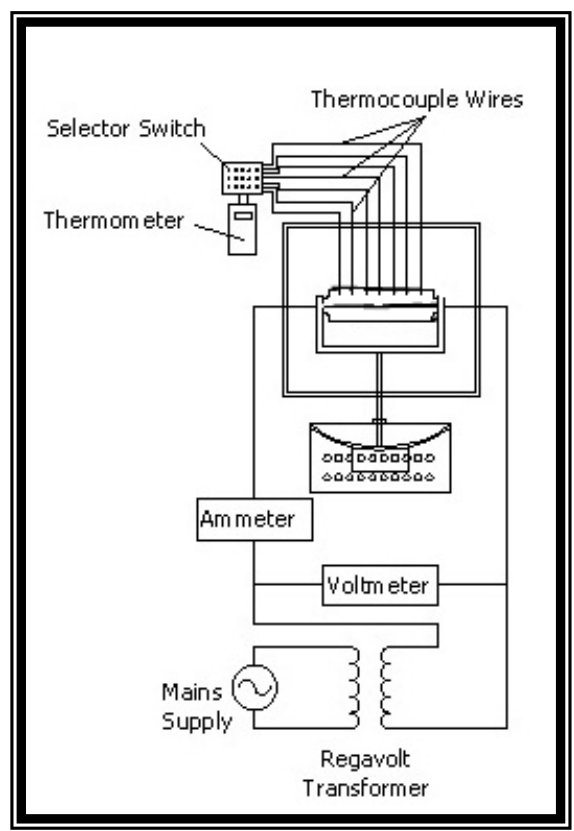

Figure 2. Schematic diagram of the thermal and electrical circle

\section{Calculation and boundary conditions}

The convective heat transfer coefficient of the finned cylinder was calculated as follows,

$$
\begin{aligned}
& Q_{g}=I * V \\
& Q_{g}=Q_{c o n v}+Q_{\text {rad }} \\
& Q_{c o n v}=Q_{s}+Q_{\text {fin }} \\
& Q_{S}=h^{*} A_{t} *\left(T_{S}-T_{a}\right) \\
& Q_{\text {fin }}=A_{\text {fin }} \eta_{\text {fin }} h^{*}\left(T_{s}-T_{a}\right)
\end{aligned}
$$

Where Qg represents the heat generation applied to the cylinder (W), Qconv is the convection heat transfer from base with fin (W), Qrad is the radiation heat transfer generated from single fin (W), h represents the convective heat transfer coefficient without vibration $(\mathrm{W} / \mathrm{m} 2 . \mathrm{oC})$, At is the base area not occupied by fin (m2), Ta is the base temperature (oC), Ta represents the base temperature $(\mathrm{oC})$, Qs heat transfer from base unoccupied by fin (W), Qfin heat transfer from single fin $(W), n$ number of fins, $\eta$ fin efficiency of the fin.

By substitution equations $(2 \mathrm{a} \& 2 \mathrm{~b})$ in equation (Qconv)., the convection heat transfer coefficient can be expressed as,

$$
h=\frac{Q_{\text {conv }}}{\left(0.1392 * \eta_{f i n}+0.0156\right)\left(T_{s}-T_{a}\right)}
$$

$\eta_{\text {fin }}=\frac{\tanh (m H)}{m H}$

Where

$m=\sqrt{\frac{h^{*} p}{k^{*} A}}$

The temperature at the hot surface measured by eight thermocouples were averaged to obtain the hot surface temperature.

The vibration speed (Uv) and amplitude of vibration can be calculated from following equations:

$\left.\begin{array}{l}U_{v}=a * f \\ a=\frac{a c c}{(2 * \pi * f)^{2} * \sqrt{2}}\end{array}\right\}$

Where $\mathrm{f}$ represents the frequency $(\mathrm{Hz})$, is the amplitude of vibration, (m), b1 is the effective length (m), acc the Acceleration of vibration, (m).

It is possible to express the vibration intensity of the following:

Vibration intensity $=a^{*} f=U_{v}$

The air properties have been calculated based on the on the average value of fin cylinder surface temperature and ambient temperature:

$T_{f}=\frac{T_{a}+T_{s}}{2}$

Where $\mathrm{Tf}$ is the film temperature (oC). An experimental study has been carried out using air as a working fluid to show the effect of forced vertical vibration on the heat transfer coefficient of the longitudinally finned cylinder. On each test, the 
inclination angle was set and heat and vibration were applied in the specified heat flux and frequency. As mentioned, the heat flux was varied between $500 \mathrm{~W} / \mathrm{m} 2$ to $1500 \mathrm{~W} / \mathrm{m} 2$.

\section{Result and discussion}

\subsection{Effect of Vibration Intensity on Heat Transfer} coefficient
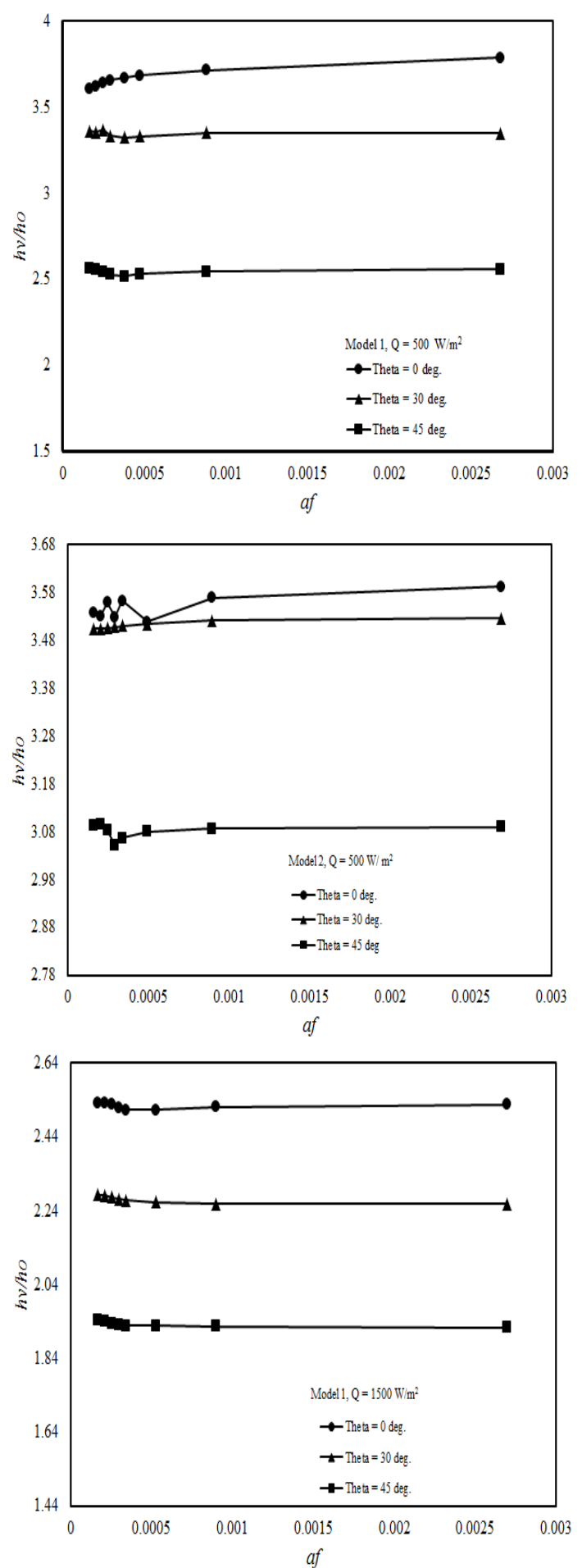

The range of vibration frequency using as all models at a horizontal angle $\left(\theta=0^{\circ}\right)$ from $2 \mathrm{~Hz}$ to $16 \mathrm{~Hz}$, and the ranged of heat flux from $500-1500 \mathrm{~W} / \mathrm{m}^{2}$ as shown in figure 3 , where ho represents the heat transfer coefficient calculated without vibration, $h v$ represented the convective heat transfer coefficient under vibration effect.
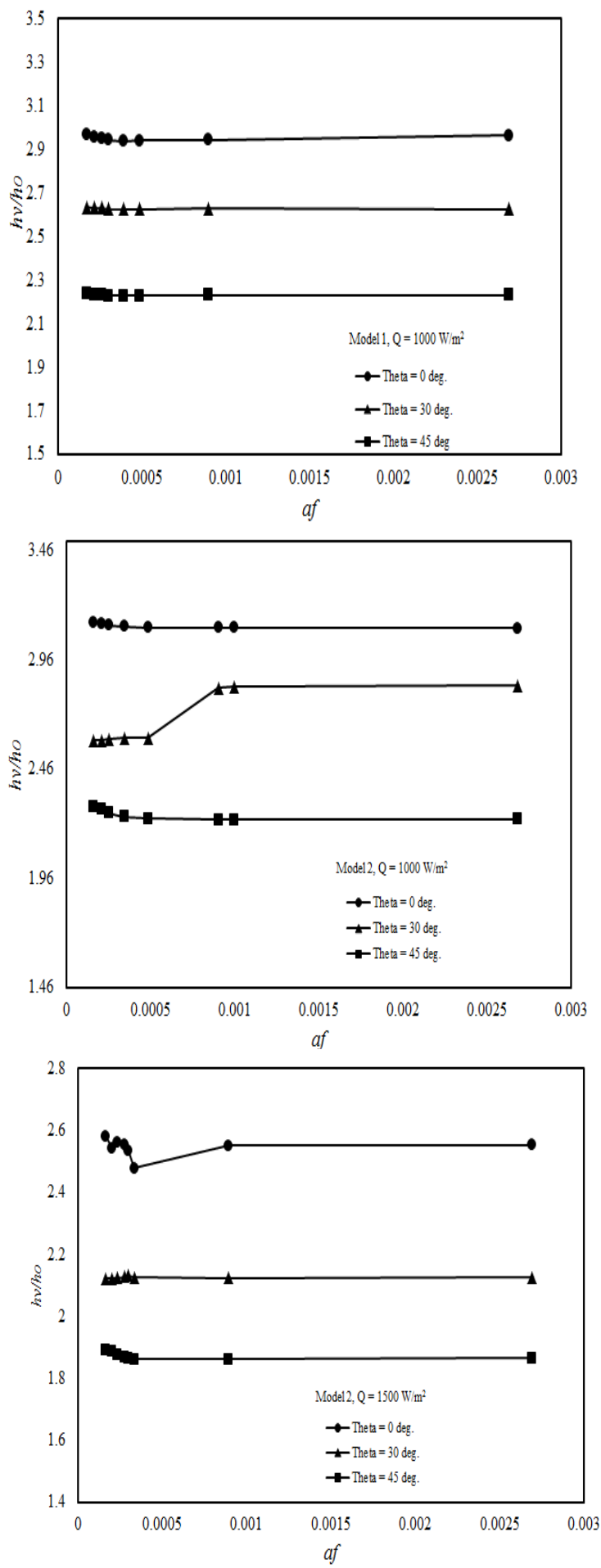

Figure 3 Shown the effect $(a f)$ on the $(h v / h o)$ for the test rig and different angle 
Figure 3, indicate that the increasing of vibration intensity leads to an increase in the heat transfer coefficient for all models at the horizontal position $(\theta=$ $00)$. Because of the vibration in horizontal position, added vibration velocity (Uv) has been generated, which is added to the main buoyancy force, and this increase the heat transfer coefficient ratio. Therefore, can deduce that in case of horizontal angle (0o), the buoyancy force component will dominate. Moreover, the fins work as paths of convection currents. Both, the main and secondary fluid flow is in the same direction, have attributed this increasing in heat transfer coefficient.

In case of model inclination at angles 300,450 , the vibration velocity (Uv) leads to turbulence in the thermal boundary layer contiguous to model surface, and then producing an additional resistance, which cause decreased of heat transfer coefficient. On the other hand, the buoyancy force component is clearly dominated, also the fins has been work as an obstruction of convection currents, which resist the fluid flow paths and eventually leads to heating of fins and decreasing the heat transfer coefficient.

Figure 3, also shows that when forced vibration occurred, the heat transfer coefficient ratio (hv/ho) at heat flux $500 \mathrm{~W} / \mathrm{m} 2$, and horizontal angle (0o) at the model (1) were $(8 \%)$ greater than those for the $(300)$ and exceeds that of the (45o) from the zero angle by $(30 \%)$. The heat transfer coefficient ratio (hv/ho) values at heat flux $500 \mathrm{~W} / \mathrm{m} 2$, of longitudinal finned cylinders in $(0 \mathrm{o})$ angle are about $(1.5 \%)$ greater than those for the (30o) and exceeds that of the (45o) from the zero angle by $(13.5 \%)$ at the rectangular fins model. From the results in figure 3 , it is clear that the heat transfer coefficient ratio increased when the vibration intensity increasing.

The results of this study showed that the heat transfer coefficient ratio (hv/ho) is improved and enhanced in model (1), where the angle of the longitudinal finned cylinders $(\theta=0 \mathrm{o})$ with triangular cross section area comparing with model (2) with rectangular cross section.

\section{Conclusions}

This study analysed and investigated the effect of vibrations which occurring in the radiator system and heat exchangers, including the external vibration effect on the heat transfer coefficient. Based on the experimental results in the previous section, the effect of the vertical oscillation on the heat transfer coefficient is illustrated. The vibration intensity affected on the heat transfer coefficient ratio (hv/ho) of horizontal angle $0 \mathrm{o}$ at the model 1, where its values increased by $8 \%$ and $30 \%$ compared with angle $30 \mathrm{o}$ and $45 \mathrm{o}$, respectively. Also the heat transfer coefficient (hv/ho) values of longitudinal finned cylinders in angle $0 \mathrm{o}$ increased $1.5 \%$ compared with the angle $30 \mathrm{o}$ and $13.5 \%$ at the angle $45 \mathrm{o}$ at model 2 . From all the results in this study, the significant effects of external vibration on fins cylinder performance and its effects on the heat transfer coefficient should be considered.

\section{References}

1. M.C.Charrier Mojtabi , Y.P.Razi , K.Maliwan and A.Mojtabi,Int. J. Heat and mass transfer, (2003).

2. R. H. Keil and M. H. I. Baird, Int. J. Heat and mass transfer, (2003).

3. B. S. Kim, D. S. Lee, M. Y. Ha, and H. S. Yoon, Int. J. of Heat and Mass Transfer, 51, pp. $1888-$ 1906, (2008).

4. A. M. Zaki, S.K. EL-Kassim, 11th International Conference on Nuclear Engineering, Tokyo, JAPAN, April 20-23, (2003).

5. Y. H. Lee, D. H. Kim, and S. H. Chang, Nuclear engineering and design 1, PP. 47-58,(2004).

6. Mujumdar, Arun S., 4th ed. Handbook of industrial drying. CRC Press, (2014). 